\title{
Los Nudos del Género. Apuntes para la Formación Ética de Educadoras y Educadores
}

\author{
The Knotty Question of Gender. Notes for the Ethical \\ Formation of Educators
}

\author{
Isabel Carrillo Flores * \\ Universidad de Vic-Universidad Central de Cataluña
}

\begin{abstract}
Las desigualdades derivadas de la diferencia sexual tienen poca presencia en la formación de educadoras y educadores. En los contextos de formación universitaria se constata que la creación de las unidades de igualdad y de centros de estudios de mujeres y de género han sido acciones positivas, pero no han eliminado plenamente la brecha entre los discursos y las prácticas. Se pone de manifiesto que tanto por lo que se hace, como por lo que no se hace, las universidades pueden contribuir a reproducir una cultura opuesta a la justicia social. En estas páginas se defiende que quiénes emprenden su caminar por la educación necesariamente han de vivir procesos formativos que muestren la verdad de los datos, puntos visibles del iceberg que hay que aprender a mirar sintiendo; que profundicen en la comprensión crítica de las realidades locales y globales aprendiendo a desvelar el currículum oculto del género que silencia las experiencias de desigualdad y negación de derechos; que promuevan el aprender a idear $\mathrm{y}$ a vivir acciones educativas transformadoras que desatan los nudos del género y que fomenten deseables éticas aplicadas que digan no a las situaciones de inequidad. La educación no sólo es ocupar un espacio, sino que como derecho también refiere a ser persona reconocida y valorada en el mismo. Sin embargo, los datos muestran que la educación no es vivida plenamente por las niñas.
\end{abstract}

Descriptores: Universidad, Enseñanza superior, Docente, Ética, Discriminación sexual.

Inequalities derived from sexual difference have little presence in the training of educators and educators. In the contexts of university formation, it is noted that the creation of equality units and women's and gender studies centers have been positive actions, but they have not completely eliminated the gap between discourses and practices. It becomes clear that both what is done and what is not done, universities can contribute to reproduce a culture opposed to social justice. In these pages it is argued that those who undertake their walk through education must necessarily live formative processes that show the truth of the data, visible points of the iceberg that must be learned to look at feeling; to deepen the critical understanding of local and global realities by learning to unveil the hidden gender curriculum that silences experiences of inequality and denial of rights; that promote the learning to devise and to live transforming educational actions that untie the knots of the genre and that they promote the applied ethical ethics that say no to the situations of inequity. Education is not only occupying a space, but as a law also refers to being recognized and valued person in it. However, the data show that education is not fully lived by girls.

Keywords: University, Higher education, Teacher, Ethics, Gender discrimination.

*Contacto: isabel.carrillo@uvic.cat

ISSN: 2254-3139

www.rinace.net/riejs/

revistas.uam.es/riejs
Recibido: $\quad 15$ de abril 2017

$1^{\text {a }}$ Evaluación: 13 de julio 2017

$2^{\text {a }}$ Evaluación: 29 de agosto 2017

Aceptado: 1 de septiembre 2017 


\section{Ausencias de los discursos de género en las universidades}

El feminismo no es sólo una teoría ni tampoco un movimiento, ni siquiera una política experta. Siendo todo eso, ha sido y es también, lo digo a riesgo de repetirme, una masa de acciones no dirigidas, a veces en apariencia pequeñas o poco significativas. Cada vez que una mujer individualmente se ha opuesto a una pauta jerárquica heredada o ha aumentado sus expectativas de libertad en contra de la costumbre común, se ha producido y se produce lo que podríamos llamar un infinitésimo moral de novedad.

El feminismo también ha sido y es esa suma de acciones contra corriente, rebeldías y afirmaciones, que tantas mujeres han hecho y hacen sin tener para nada la conciencia de ser feministas. Esto es, tales acciones se realizan sin la conciencia de una voluntad común. Creo que en este momento y en esta tercera ola del feminismo al que pertenecemos, que es la que se abre a un tercer milenio, las mujeres pueden ser ya capaces de forjar una voluntad común relativamente homogénea en sus fines generales: conservar lo ya hecho y seguir avanzando en sus libertades. (Valcárcel, 2008, p. 331)

Aunque los informes de organizaciones gubernamentales y no gubernamentales sobre las desigualdades que viven las mujeres desde la infancia sigan mostrando los grandes nudos del género aún sin resolver, y aunque persisten las resistencias a reconocer los movimientos feministas como aportaciones significativas para la construcción de sociedades democráticas y el bienestar de todas las personas, lo cierto es, como señala Valcárcel (2008), que los obstáculos que persisten no han paralizado el dinamismo de las acciones a contra corriente. Estas acciones de rebeldía no sólo denuncian, sino que al mismo tiempo muestran evidencias que las realidades injustas, que se justifican en la diferencia sexual, no son así porqué es normal y natural, sino que lo son intencionalmente para mantener sociedades asimétricas y jerarquizadas.

Perpetuar el orden establecido es interés de grupos que quieren continuar moviendo los hilos del poder para afirmar su posición de privilegio y de dominio, aunque sea a costa de establecer relaciones de subordinación, de marginación y de opresión de las mujeres. En oposición, las acciones a contra corriente expresan una necesaria conciencia común de que las realidades no están determinadas, que pueden cambiarse para ser otras distintas reconocedoras de la dignidad y los derechos de las mujeres, aunque para ello habrá que desvelar el entramado de las resistencias que se oponen a las transformaciones justas.

\subsection{La brecha entre el discurso normativo y la formación universitaria}

En los contextos de formación universitaria se constata que la creación de las unidades de igualdad y de centros de estudios de mujeres y de género han sido acciones positivas, pero no han eliminado plenamente la brecha entre los discursos y las prácticas. Se observan disonancias no sólo entre grados diferentes, sino en el marco de un mismo grado y plan de estudios. Ello sucede a pesar de que en muchas realidades la creación de dichas unidades y centros es el resultado de la aprobación de leyes de igualdad, pero ocurre que frecuentemente se ignora el conocimiento de sus contenidos y las obligaciones que conllevan, entre otras la transversalidad y abordaje específico del género en los planes de estudio de grado y postgrado.

De forma bastante generalizada las universidades no cumplen regularmente la normativa jurídica. Ante el no cumplimiento de la ley parece no ocurrir nada, o más bien lo que ocurre es la tolerancia de la propia comunidad universitaria reforzada por la 
permisividad política. En el caso español, el 22 de marzo de 2017 fue el décimo aniversario de la Ley Orgánica 3/2007, de 22 de marzo, para la igualdad efectiva de mujeres y hombres (BOE, 2007). Algunas noticias de diarios digitales del mismo día de celebración recogían en sus titulares expresiones relativas a lo poco que celebrar (Público), el cambio que nunca llega (El País), o lagunas en la ley de igualdad (La Vanguardia). Expresiones que bien pueden trasladarse a la realidad de las universidades, como también de las escuelas, institutos, centros de formación de personas adultas y otros centros de formación. En educación la igualdad tiene aún mucho camino que recorrer. No todo está dicho y está hecho por incorporar términos como coeducación, equidad, o inclusión -aunque éste último no es un término habitual en los discursos sobre diferencia sexual-. Los nudos del género persisten en los contextos formativos de las universidades de las sociedades actuales, los estudios lo confirman.

La baja presencia de contenidos sobre igualdad y género en las universidades ha sido constatada en el estudio, La perspectiva de gènere a la docència $i$ a la recerca a les universitats de la Xarxa Vives (2017), que evidencia que sólo un 17\% de los grados universitarios incorpora asignaturas específicas en materia de género, y de éstas el $61 \%$ son optativas. En el caso de la formación de educadoras y educadores, en el XXXV Seminario Interuniversitario de Teoría de la Educación se presentó la ponencia Educación y género en la formación docente en un enfoque de equidad y democracia que puso de manifiesto que el articulado de la Ley 3/2007, que dispone la introducción de contenidos relacionados con la igualdad y la no discriminación en la formación universitaria, no se está cumpliendo. Indicar que en el Capítulo II, de la mencionada ley, relativo a la acción administrativa para la igualdad, se establecen disposiciones sobre la formación. En concreto en el artículo 25, referente a la igualdad en el ámbito de la educación superior, se dispone el fomento de la enseñanza y la investigación sobre el significado y alcance de la igualdad entre mujeres y hombres, la inclusión de enseñanzas en materia de igualdad, la creación de postgrados específicos, y la realización de estudios e investigaciones especializados en la materia.

Sin embargo, parece que las realidades se resisten a los cambios que indican las normativas. En la ponencia se afirma que las universidades continúan siendo escenarios en los que el espejismo de la igualdad está muy presente $\mathrm{y}$, como consecuencia, el discurso sobre la igualdad de género está ausente de los grados de infantil y primaria, de los másteres de formación del profesorado de secundaria y de las tesis. En la ponencia se muestran los resultados de la revisión de los planes de estudio de 44 universidades españolas que imparten los grados de educación infantil y educación primaria. De las universidades analizadas, 11 incorporan asignaturas con contenidos explícitos de igualdad de género, en 9 de ellas la asignatura es obligatoria: Diversidad y Coeducación, Universidad de Sevilla; Educación en Igualdad de Género, Universidad de Alicante; Educación para la promoción de la igualdad entre los géneros, Universidad de Las Palmas de Gran Canaria; Identidad de Género y educación, Universidad de Santiago de Compostela. En otras 5 universidades en algunas asignaturas se recoge la palabra igualdad en su título: Educar para la igualdad y la ciudadanía, Universidad Autónoma de Madrid; Diversidad cultural, derechos fundamentales, igualdad y ciudadanía, Universidad Pública de Navarra; Educación para la paz e igualdad, Universidad de Valladolid (Ayuste et al., 2016). 


\subsection{En educación no todo es tolerable}

Los resultados de los estudios confirman una anecdótica presencia que se traslada a otros centros y etapas educativas. En la práctica del oficio de educar las maestras y los maestros tampoco expresan un gran interés por una formación específica, quizás en parte a causa de una formación inicial que no ha hablado de ello y no ha despertado la necesidad. En parte debido a que en las escuelas también opera el espejismo de la igualdad y de la coeducación -que no es igual a escuela mixta-; un espejismo que no permite ver que las desigualdades de género que se viven fuera de los centros entran en los espacios escolares, al mismo tiempo que dentro de los centros operan mecanismos que mantienen los nudos del género provocando vivencias de inequidad. Los itinerarios diferenciados en centros segregados por sexos tampoco contribuyen a avanzar en la construcción de sociedades justas no divididas, dificultando al mismo tiempo la vivencia de ciudadanías inclusivas.

Las ausencias en los planes de estudio de asignaturas y contenidos sobre igualdad y género se acompañan, entre otros problemas no resueltos, de la escasa inclusión de referencias bibliográficas de aportaciones de mujeres en asignaturas no específicas; de la naturalización de la feminización de la educación; y de la ausencia del análisis específico de las situaciones de desventaja que viven las mujeres a nivel global, como es ejemplo una mayor negación del derecho a la educación de las niñas. Estos hechos se refuerzan mediante los usos de un lenguaje que olvida la diferencia sexual. La consecuencia es que vivimos un exceso de tolerancia ante realidades que deben despertar rechazo y no tolerarse.

Como valor deseable la tolerancia "es la virtud indiscutible de la democracia", es el "respeto a los demás, la igualdad de todas las creencias y opiniones, la convicción de que nadie tiene la razón absoluta", es "apertura y generosidad" que se opone a las actitudes de intolerancia que conducen "al totalitarismo". Pero también es cierto que la tolerancia "no es ni debe ser lo mismo que la indiferencia" y, en este sentido, "es lícita la intolerancia con los puntos de vista distintos" siempre que sea para prevenir el daño a otras personas o al propio yo (Camps, 1990, pp. 81-102).

En la formación de educadoras y educadores hay que respetar la diferencia sexual y hay que rechazar la tolerancia ante prácticas que reproducen las desigualdades entre mujeres y hombres, porque quienes se comprometen con la educación no pueden ser cómplices de injusticias. De ahí la necesidad de tener presentes unos mínimos valorativos.

\section{Mínimos para una formación ética de educadoras y educadores}

La Cenicienta tenía tantas, tantas ganas de ir a la fiesta... que al final lo consiguió. Pero se puso tan ansiosa... que a la mañana siguiente no se acordaba de nada (llegó a las 12, pero a las 12 del día siguiente)

Cuidado es peligroso llegar a estos estados de inconsciencia

Pero ahi estaban esos dos señores, con el zapato de cristal de tacón de palmo y de punta... esperando para que se lo probara. Al principio no le cabía en el pie, pero apretó y apretó hasta que le "cabió" y metió la pata... iporque se tuvo que casar con el príncipe! (Nunila López, 2009, p. 2) 
Las ausencias muestran los olvidos, los vacíos, lo que intencionalmente no está, pero al mismo tiempo abren la posibilidad de proyectar los principios éticos que podrían formar parte de los planes de estudio de las universidades, de todas, porque todas tienen el deber de ser responsables socialmente y actuar en coherencia. Es así que en la concreción de la formación de educadoras y educadores no puede adoptarse una supuesta posición de neutralidad ante el género, por qué no hay educación neutral en el sentido que "no hay práctica educativa que no sea política; no hay práctica educativa que no esté envuelta en sueños; no hay práctica educativa que no involucre valores, proyectos, utopías. No hay entonces, práctica educativa sin ética" (Freire, 2003, p. 43).

\subsection{Una formación que proyecta procesos éticos}

Es en una orientación política y ética que la formación universitaria en el ámbito de la educación, consciente de la normativa de género, tiene que atender como mínimo a tres finalidades que contemplan: el aprender a mirar sintiendo; el aprender a pensar ideando; y el aprender a actuar éticamente. Los sentidos de tales finalidades se exponen a continuación.

\subsubsection{Aprender a mirar sintiendo}

Con esta expresión se hace referencia a educar para agitar, para emocionarse, para no quedarse indiferente, para contribuir a descubrir la verdad de las desigualdades promoviendo procesos para aprender a mirar y a sentir las imposiciones normativas del género que, como se narra en el cuento de La Cenicienta que no quería comer perdices, sólo permiten usar zapatos de tacón de palmo y de punta que no te dejan poner la espalda recta, porque te caes, el cuerpo se va inclinando y por la espalda se deslizan todas las ideas e ilusiones (López, 2009). La educación no puede contribuir a doblegar el ser de cada persona, de cada mujer, negando la autonomía del yo que se quiere ser.

Aprender a ver las realidades injustas que viven las mujeres de forma globalizada debe ser finalidad de la formación en educación. Lo debe ser para despertar el deseo de mirar la realidad, pero no únicamente para contemplarla, sino para saber observarla, cuestionarla cuando se descubren prácticas injustas y admirarla cuando se observan prácticas de justicia. Es un aprender a percibir desde diferentes ángulos y perspectivas y, en este proceso, aprender a ver su complejidad realizando miradas panorámicas y también focalizadas, lejanas y cercanas que posibilitan descubrir el todo y las partes, la globalidad y los detalles, lo que es real y lo que es inventado. Aprender a mirar para quitar los velos y descubrir lo que es y lo que no es, o es únicamente apariencia, y en este mirar darse cuenta de que es el propio yo el que participa en la de-codificación de la realidad y en la conformación libre de imágenes sobre la misma, rompiendo las visiones ya dadas, limitadas y excluyentes.

Mirar es participar de lo que se ve, experimentar que no se está al margen de la realidad que nos rodea, sino saber que formamos parte y al hacerlo no únicamente se mira, sino que también se siente. El aprender a mirar despierta el aprender a sentir cuando la educación hace hincapié en la sensibilidad, en el yo sensible. En los procesos de formación de educadoras y educadores se debe promover el deseo de aprender a percibir y notar sensaciones, a experimentar a través de los sentidos, sabiendo que es en la relación educativa que se estimula la sensibilidad y las disposiciones afectivas para dejarse tocar sensiblemente por la realidad. Un aprender a estar en alerta, en contraposición a un ser ajeno al mundo del que se forma parte. Un aprender a saber 


\section{Carrillo}

emocionarse y no quedarse indiferente, sintiendo que se participa en la construcción autónoma de los propios sentimientos, pues se aprende a reconocerlos, a expresarlos y a compartirlos.

Aprender a mirar sintiendo es un aprendizaje ético no sólo emocional, sino al mismo tiempo es racional, afectivo y cognitivo, consciente y concientizador que se acompaña del aprender a pensar e idear.

\subsubsection{Aprender a pensar ideando}

Es éste un proceso que pone en el centro una educación para preguntar, para saber, para tomar conciencia y concientizarse. Junto al aprender a ver hay que aprender a desvelar lo que queda oculto a través de procesos de comprensión crítica, de análisis de los mecanismos que operan en sociedades clasificadoras de la diferencia sexual y negadoras de derechos para las mujeres. Comprender para aprender a decir BASTA a las opresiones que viven las mujeres, y BASTA a las opresiones, que vive el propio yo, como narra la protagonista del cuento de la Cenicienta que no quería comer perdices (López, 2009).

Promover los anteriores procesos requiere de una educación del aprender a pensar que es generadora de espacios para reflexionar, para despertar el deseo de descubrir, de conocer y de poner en relación ideas diversas. Es a través de estos procesos que cada mujer y cada hombre, con voz propia, aprende a pensar la realidad próxima y lejana, el yo y el mundo, el pasado y el presente. Pensar es participar de la realidad aprendiendo a preguntarse y preguntar, a analizarse y analizar el mundo, a valorarse y valorar la exterioridad. Es un aprender a pensar querido que se da a través de la razón dialógica y sensible que permite conocer, visibilizar y relacionar pensamientos diversos, al mismo tiempo que se cuestionan las concepciones que acompañan el yo. Este proceso de pensar participativo incide en la toma de conciencia y la comprensión humana necesarias para despertar el deseo de idear.

La educación es también creación, por lo que el aprender a idear forma parte de un hacer educativo que es crítico y movilizador, en el sentido que la educación no se puede entender como socialización reducida a la adaptación acrítica que se traduce en asumir las concepciones y prácticas ya establecidas. La formación debe motivar el aprender a participar en la creación de nuevas ideas para definir proyectos utópicos pero posibles. La utopía no es lo irrealizable, es horizonte que debe servir para construir el ahora como contrapunto a la reproducción acrítica. Hay que formar para que a través de la educación se motive la ideación creativa de la realidad que se quiere vivir.

En el proceso de ideación convergen y se entrelazan los imaginarios que se van gestando en el diálogo constante del mirar, del sentir y del pensar para concebir y dibujar formas de vida que son expresión de valores éticos que orientan el actuar.

\subsubsection{Aprender a actuar éticamente}

Este es un aprendizaje que requiere el educar para movilizarse, para transformarse y transformar, para impulsar procesos reales de cambio a través de propuestas educativas que motivan, junto al aprender a idear, el aprender a vivir éticas aplicadas. Una formación que se focaliza en el aprender a desatar los nudos del género, incidiendo de forma especial en el derecho a la educación de las niñas. Decir BASTA es un primer paso que se sigue de otro que es pensar en COSAS BONiTAs, que lleva a tener ganas de empezar a caminar. Es así que la Cenicienta “dejó al príncipe (a pesar de que cuesta 
mucho dejarlos, es tan difícil que a veces repites 203 príncipes más). También "dejó los zapatos y las perdices" y descubrió "la danza libre, que no es tan libre pero que te hace sentir libre", pues en esta danza "da igual que calces un 42, que peses 90 kilos, que midas 1,92 o que tengas 80 años” (López, 2009, pp. 5-9). La ideación toma vida en la acción, haciendo, aprendiendo a vivir humanamente.

Es en esta orientación que la educación es proyección y acción, es encaminar el actuar, es la vivencia de lo que se siente y se piensa, de lo que se proyecta como éticamente deseable. La formación de educadoras y educadores deberá ser discurso y práctica que motiva el deseo de aprender a actuar desde la propia autonomía moral. Un actuar virtuoso en el entorno próximo, pero contextualizado en una ética planetaria. Un actuar que se fundamenta en perspectivas pedagógicas que permiten desatar los nudos del binomio sexo-género que establece la normalidad, lo que es normal enfrentado a lo que no es normal porque se le atribuyen carencias, defectos o errores. Para ello se precisa incorporar no sólo los discursos sobre la diferencia y la deconstrucción de los géneros, sino también propuestas educativas que amplíen los significados de la inclusión. La educación inclusiva será educación para la equidad si se es plenamente consciente de la interrelación de las diferencias en el análisis de las desigualdades y en las propuestas para erradicarlas, y si tales procesos convergen en la acción, es decir, en la expresión de verdaderas éticas aplicadas.

\subsection{Una formación pedagógicamente no neutral}

Una formación ética desveladora de las desigualdades de género, y creadora de formas de vida justas, es una formación pedagógicamente no neutral que adopta un enfoque de derechos y de justicia en los contenidos de la formación superior de educadoras y educadores. En esta perspectiva los derechos humanos, y el derecho a la educación como derecho humano, deberán ser ejes articulares de la formación, no sólo para conocerlos, sino para aprender a decir no a una educación negada. La educación es derecho moral y jurídico fundamental, tiene un carácter universal, indivisible e interdependiente, y es consustancial a la persona (Añón, 2014; Rodríguez, 2011).

En el documento de trabajo Relacions de poder, violències i altres formes de relació abusiva. La transversalitat del gènere (Carrillo, 2016), realizado en el marco del Programa de Millora i Innovació en la Formació de Mestres del Consell Interuniversitari de Catalunya i la Secretaria d'Universitats i Recerca, se proponen contenidos, principios y prácticas que pueden orientar los análisis de los planes de estudio de formación docente en un enfoque ético, de derechos humanos y de justicia social. Esta propuesta, abierta y flexible, considera que en la formación de educadoras y educadores deberían incorporarse, entre otros posibles, bloques de contenido referentes a dimensiones de la educación que se complementan entre sí: la dimensión relacional; la dimensión de la inter-relación de las diferencias; la dimensión política y ética; y la dimensión práctica.

\subsubsection{La dimensión relacional de la educación}

La dimensión relacional supone incluir contenidos sobre las relaciones de poder entre las personas en una doble orientación. Por una parte, como relaciones que pueden ser abusivas y hacer uso de violencias. Por otra parte, como reconocimiento que "el poder no sólo es el que se nos impone y nos obliga a subordinarnos, sino también el que poseemos y muchas veces desconocemos y que puede orientarse individual y colectivamente a la 


\section{Carrillo}

transformación de las subordinaciones" (Olivera, 2004, p. 21). Opresión y empoderamiento son conceptos claves en la formación de educadoras y educadores de todas las etapas y ámbitos de la educación.

Al mismo tiempo, en relación a la dimensión relacional también hay que incorporar contenidos sobre un saber hacer docente ético que acompañe el aprender a convivir de forma harmónica y sea coherente con las diferentes generaciones de derechos humanos, así como los valores que emanan de los mismos (Pérez, 2006). Habrá que educar atendiendo a dos procesos interconectados. Por una parte, para conocer las distintas generaciones de derechos humanos y comprender su carácter dialéctico, es decir, la necesidad de ampliarlos y revisarlos a la luz de nuevas realidades. Por otra parte, para desvelar que tales derechos no son vividos de forma plena por las mujeres, pues en sus vidas relacionales se encuentran limitadas en sus libertades -la libertad remite a la primera generación de derechos, los civiles y políticos-; experimentan la desigualdad -la igualdad refiere a la segunda generación de derechos, los sociales, económicos y culturales-; y viven en soledad los graves efectos de las guerras y de los entornos no sostenibles -la solidaridad se vincula a la tercera generación de derechos relativos a vivir en sociedades en paz y sostenibles-.

Lo expuesto lleva a pronunciarse respecto al enfoque de derechos que hay que adoptar en la formación de educadoras y educadores, pues ésta debería ser una formación de solidaridades con las niñas, las adolescentes, las jóvenes, las adultas, es decir, con todas las mujeres en su diversidad, exigiendo la igualdad en las diferencias y el derecho a la libertad para ser y ser protagonistas de la propia vida.

\subsubsection{La dimensión de inter-relación de las diferencias}

Considerar que las personas son diversas, y al mismo tiempo reconocer que hay diferencias que son utilizadas para justificar discriminaciones no justas, es contenido obligado en la formación. Esto supone corregir en los planes de estudio el hecho de hablar de la diferencia sexual sólo como un dato fijo -biológico-, porque no lo es. La diferencia sexual es "un dato que impregna la relación de cada ser humano con la realidad, sexuándola y, al hacerlo, sitúa a mujeres y hombres en posiciones desiguales" (Rivera, 2005, p. 15). Este dato se asocia a un género que prescribe como ser y cómo actuar, pero no únicamente, sino que es un dato que en interacción con otras prescripciones que imponen la clase, la etnia, la cultura, la edad, el territorio que se habita..., obligan a adoptar identidades no libremente deseadas. Cómo es concebida y vivida la diversidad es contenido imprescindible de una formación que debe ser sensible a la misma en sociedades que no son homogéneas.

Hablar de diversidad conlleva la reflexión sobre la duplicidad de significados de las diferencias. Por una parte, hay diferencias que son deseables porque expresan el yo singular y su autonomía moral. Por otra parte, hay diferencias no deseables, son aquellas que se establecen para provocar situaciones de desigualdad. La diferencia sexual, la de las mujeres, y las prescripciones del género femenino, continúan operando como detonante de tratos diferenciales que provocan vivencias de desventaja y exclusión.

Las desigualdades que se sustentan en el binomio sexo-género no pueden estar ausentes en la formación de educadoras y educadoras, como tampoco pueden estar ausentes contenidos sobre la democracia como el contexto político y de convivencia más deseable para la expresión de diferencias deseables y de ciudadanías inclusivas. 


\subsubsection{La dimensión política y ética de la educación}

La educación es ideológica, lo es tanto en su función de reproducción como en su función de transformación. Es por ello que la dimensión política y ética de la educación es otro de los contenidos a incorporar en la formación, alejando la sombra de falsas neutralidades. No se puede mentir acerca de los principios ideológicos que impregnan y acompañan el hacer educativo. La educación, en sí misma, es valor o contravalor en función de los principios políticos que se adopten. Se puede tener la orientación de educar en valores proactivos -valores deseables que hay que despertar y estimular como los derechos planetarios, la libertad, la autoestima, la interioridad...-, o educar en valores reactivos -contravalores que se promulgan en exceso y habría que eliminar como el individualismo, la falta de compasión, la competitividad, la exterioridad...- (Cortina, 2010).

Esta dimensión contempla el compromiso ético docente, así como la responsabilidad individual y colectiva, de un oficio que bien puede contribuir a reproducir desigualdades o a transformarlas. Incorporarla en la formación de educadoras y educadores va a permitir analizar cómo el conocimiento puede tener poder transformador, es decir, cómo la ampliación del saber y el saber otros saberes puede contribuir a promover cambios justos, que lo son cuando las mujeres no quedan excluidas de los mismos.

Conocer el valor transformador de la educación también es necesario para una formación que ha de substraerse del pensamiento único paralizador.

\subsubsection{La dimensión práctica de la educación}

De la abstracción a la vivencia de éticas aplicadas. Éste debe ser el contenido articulador del currículum en la formación docente. La dimensión práctica de la educación refiere a la necesidad de abordar la formación de educadoras y educadores desde una perspectiva compleja, y no desde el aislamiento de asignaturas que se compartimentan en espacios impermeables a conocimientos que se consideran ajenos, cuando no menores, o no necesarios en la formación, como ocurre con los contenidos relativos a los feminismos y el género. La formación práctica necesita adoptar el reto de la complejidad, que no es sólo abrir las fronteras entre las disciplinas para conformar un pensamiento integrador, sino abordar cómo transformar lo que genera las fronteras, es decir, los principios organizadores del conocimiento (Morin, 2001).

En el sentido apuntado los discursos de género deben articularse transversalmente para formar parte de los otros ámbitos de conocimiento, pero no únicamente. Al igual que se defiende una formación no coactiva ni opresora, sino empoderadora, los saberes que aportan los feminismos y las teorías del género deben ser liberados del olvido y de la subordinación para ser empoderados incorporándolos, con identidad propia, en los planes de estudio. La formación práctica ha de proyectar las formas de ser plurales, sólo así podrá ir emergiendo ese "ir siendo, aquí y ahora, las mujeres que queremos ser", mujeres "con capacidad democratizadora en el mundo", mujeres no silenciadas que actúan por sociedades justas (Lagarde, 2000, p. 27). La formación que se proyecta ha de acompañar el ir aprendiendo a descubrir y valorar los saberes negados, motivar el deseo de conocerlos y, al mismo tiempo, querer revisar críticamente el canon del conocimiento que se transmite como único válido. 


\section{Carrillo}

\subsection{Eliminar las resistencias de género para una formación éticamente transformadora}

Las resistencias a cambios en las concepciones, en los contenidos, en los materiales, en las formas organizativas, en los tiempos... de los planes de estudio de la formación docente son palpables; eliminarlas es urgente, siendo necesario el compromiso no sólo individual sino de toda la comunidad universitaria.

Con objeto de contrarrestar las resistencias habrá que activar diversos procesos. Por una parte, establecer espacios de reflexión compartida del profesorado de los grados de educación que permitan analizar los impactos de género de los planes de estudio. Por otra parte, y atendiendo a los datos que ofrece el análisis de la realidad, delimitar los cambios a realizar, entre otros, en los marcos ideológicos, en la articulación de teoría y práctica y en el currículum.

\subsubsection{Resistencias de los marcos ideológicos de género}

Eliminar estas resistencias es un proceso que va a requerir la voluntad de no ocultación. Es necesario explicitar los marcos ideológicos que inhiben o favorecen la inclusión de las desigualdades de género, de los feminismos y de la teoría de género como instrumentos de análisis en los planes de formación de educadoras y educadores.

Los equipos de los propios centros deben estar dispuestos a la autocrítica con la finalidad de identificar los elementos de la cultura androcéntrica, las relaciones patriarcales que se perpetúan, los instrumentos sexistas que están presentes en las normativas, en la gestión, en la organización, en el currículum, en los espacios, en los tiempos..., así como en las actitudes y en el hacer docente. Se deberán identificar tales resistencias con el propósito de diseñar acciones conjuntas para eliminarlas de los planes de estudio y, en contraposición, proyectar otras ideologías con la finalidad de poner en práctica una formación que, de verdad, tenga una orientación éticamente transformadora.

\subsubsection{Resistencias de la teoría desvinculada de la práctica}

La formación en educación no puede contemplar únicamente la incorporación de los contenidos de género en las áreas de fundamentación pedagógica, sociológica, psicológica, entre otras materias de fundamentación y comprensión holista de la realidad, sino que estos contenidos han de impregnar la totalidad del currículum formando parte de parte de las áreas y didácticas específicas de los grados de educación, en todos los cursos.

Eliminar las resistencias de exclusión de los contenidos de género requiere, de forma especial, atender a las prácticas ya que pueden permitir vincular el conocimiento teórico que se analiza en las diferentes áreas de conocimiento y en los seminarios específicos de prácticas con la aplicación. La teoría debe traducirse en acciones educativas que pueden realizarse en el propio contexto universitario, en el entorno comunitario y durante la estancia de prácticas en las escuelas u otros centros educativos.

La eliminación de estas resistencias requiere no olvidar el recorrido y las tensiones de género que pueden vivirse en las transiciones formativas de las universidades a la comunidad y a las escuelas y del entorno a las universidades. 


\title{
2.3.3. Resistencias del hermetismo curricular
}

Una tercera resistencia que hay que eliminar es la tendencia al hermetismo de los planes de estudio que dificulta la incorporación de contenidos excluidos de los mismos. Hacer frente a dicha resistencia lleva a contemplar la estructuración de asignaturas específicas obligatorias -no sólo optativas- sobre educación y género cuyos contenidos tendrán que atender a las problemáticas globales de género, así como a los problemas focalizados que se viven en las realidades próximas. La formación ha de articular los nudos del género del pasado y del presente. Debe hacerlo por la razón ética que la memoria "es imprescindible para una acción transformadora del ser humano en sus contextos sociales, políticos, económicos y culturales". Hay que fomentar una "memoria obligada", demasiado ausente de los planes de estudio, porque el derecho a la memoria es "inseparable del derecho que toda persona tiene a conocer la verdad" (Erazo, 2011, p. 13).

Junto a estos contenidos, no hay que olvidar incorporar los relativos a la teoría del género como marco interpretativo de las realidades de desigualdad; las pedagogías de la equidad y la no violencia; los lenguajes, discriminaciones y violencias; así como el enfoque de derechos humanos en la educación y la educación como derecho humano.

Por una parte, el lenguaje es contenido singular en el currículum ya contemplado en las áreas lingüísticas, pero como elemento de comunicación impregna cualquier tratamiento de contenidos de otras áreas. Por su parte, el derecho a la educación es fundamento y finalidad de toda acción formativa que atraviesa el currículum. Ambos contenidos mantienen los nudos del género, problemas aún sin resolver que requieren de acciones urgentes orientadas a la deconstrucción de los géneros. En los siguientes apartados se realizan algunas reflexiones al respecto.

\section{Las palabras y sus impactos de género, puntos visibles del iceberg}

\begin{abstract}
Mi hermana nació justo antes del crudo invierno de 1963. Nuestra madre no estaba casada por entonces. En 1963 era algo extraordinario tener un bebé si no estabas casada, asi que la madre de mi hermana era una madre soltera. ¿Qué era mi hermana? ¿Y qué era su padre? Me lo pregunto. (Una, 2016, p. 17)
\end{abstract}

La cita con que se inicia este apartado no explica una situación del pasado. Aún hoy hay madres que no son sólo madres, sino que son madres solteras, una adjetivación que mantiene un prejuicio valorativo, un nudo de género. Las palabras no sólo nombran, sino que al hacerlo atribuyen cualidades o no cualidades, es decir, aprecian o estigmatizan negativamente. Es así que, en su uso, el lenguaje aún se resiste a reconocer el ser madre que rompe con los marcos normativos del género. Además, la adjetivación que se utiliza refiere a la definición del yo mujer en base a las relaciones que se establecen entre grupos que ostentan posiciones de poder y valoración no equiparables. Esto ocurre en el ámbito público y privado, por lo que también permea los centros educativos y los mensajes que en ellos se vehiculan. Cambiar los usos del lenguaje es reto de una formación que no quiere ser sexista, sino una formación justa que utiliza las palabras para desvelar, denunciar y evaluar los impactos de género. 


\section{Carrillo}

\subsection{Desvelar la justificación falsa de lo técnicamente correcto}

Aunque pueda resultar repetitivo recordarlo, es obligado evidenciar la distorsión y ocultación de la realidad que los propios centros generan a través de prácticas diversas, como es ejemplo el uso sexista del lenguaje. No es ficción ni un hecho del pasado. No hay razón técnica que lo justifique, porque cualquier norma, método, acción que vulnera los derechos humanos debe cambiarse, y si se persiste en ello hay que desvelarlo y denunciarlo. Las educadoras y los educadores tienen el compromiso ético, el "imperativo moral", de difundir las "verdades esenciales" sobre "las cuestiones más importantes". Hay que "descubrir la verdad", pero "no con la imposición de la verdad oficial" pues ello inhibiría el "desarrollo de un pensamiento crítico e independiente" (Chomsky, 2001, p. 29).

Las desigualdades entre mujeres y hombres, que también se reproducen a través de los usos no inclusivos del lenguaje, debe ser objeto de análisis y cambios en la formación universitaria. Hay que decir la verdad para, por una parte, enseñar a aprender a percibir los puntos visibles del iceberg, y los usos de las palabras lo son; por otra parte, aprender a preguntarse por la dimensión de lo que queda oculto en un mar de aguas que se muestran opacas a nuestra mirada. La transmisión ideológica en el uso del lenguaje y el poder relacional de las palabras, de lo que se nombra o no se nombra, o de cómo se nombra, forma parte de esa esfera que se quiere mantener en el mundo de las opacidades de las normativas del género.

Los usos del lenguaje en los centros educativos, en la propia formación de educadoras y educadores, ponen de manifiesto las ambivalencias entre la reproducción y la transformación. En el Título II de la Ley 3/2007 se definen las políticas públicas para la igualdad. De los diferentes artículos del Capítulo Primero referente a los principios generales, el artículo 14.11. establece de manera imperativa la implantación de un lenguaje no sexista en el ámbito administrativo y su fomento en la totalidad de las relaciones sociales, culturales y artísticas. ¿Por qué entonces tantas resistencias a un cambio del mismo que nombre a las mujeres, a sus saberes y aportaciones enriqueciendo al propio lenguaje al mismo tiempo que amplía el conocimiento sociocultural? Si hay que educar en igualdad y para la igualdad, el lenguaje no puede ser un impedimento, y lo es cuando se usa como instrumento sexista.

También es importante usar el lenguaje para explicar que en las prácticas docentes hay personas que tienen cuidado con las palabras y actúan de acuerdo a la justicia curricular, es decir, atienden a los intereses de las personas menos favorecidas, fomentan la participación democrática, y tienen la disposición de producir más igualdad. En su hacer, el currículum contrasexista deja de ser una denominación de área aislada o de posicionamiento opuesto, para convertirse en principio de democratización de la educación (Connell, 1999). Sin embargo, se constata que son habituales otras prácticas que dan indicaciones técnicas para usar únicamente el masculino cuando se quiere nombrar a los sexos (en un plural que no es homogéneo), argumentando que esto es lo técnicamente correcto y objetivo.

Pero es falso, porque lo aparentemente técnico y correcto no es objetivo ni neutral, sino una decisión profundamente ideológica que inculca normativas de género. Así, se convierte en correcto hablar de maestros al referirse a maestras, o los estudiantes cuando son las estudiantes, ignorando que en las aulas la mayoría son mujeres. Las cifras así lo revelan. En el contexto español, los datos publicados por el Ministerio de 
Educación, Cultura y Deporte relativos al curso 2014-15 muestran que, de un total de 52.099 docentes de educación infantil, 1.238 corresponde al sexo hombres y 50.861 al sexo mujeres. En educación primaria tampoco se observa paridad, ya que, del total de 228.839 docentes, 43.317 corresponde al sexo hombres y 185.522 al sexo mujeres. Ellas, las maestras, son las más presentes en infantil y primaria; pero ellas, las maestras, siguen sin ser nombradas. Ellas, las estudiantes de maestra, aprenden pronto a no nombrarse al mismo tiempo que aprenden a nombrarse en masculino, como aprenden a nombrar a grupos de niñas y niños sólo como niños.

\subsection{Denunciar el lenguaje excluyente}

¿Por qué muchas de las profesoras y muchos de los profesores de las universidades no aplican la Ley 3/2007 de igualdad? ¿Acaso lo único que hacen es reproducir, sin cuestionar, la terminología del Real Decreto 1594/2011, de 4 de noviembre, por el que se establecen las especialidades docentes (BOE, 2011), una norma que alude al Cuerpo de Maestros que desempeñen sus funciones en las etapas de Educación Infantil y de Educación Primaria?

La normativa hace uso de la palabra maestros como genérico para nombrar a un colectivo diverso. No se comprende que una norma posterior a una Ley Orgánica de igualdad, ésta de rango superior, no se corresponda con lo que en ella se establece. Tampoco se comprende tanto silencio a las resistencias a usar el femenino. Ningún grupo debe oprimir al otro no nombrándolo, como si no existiera. Por ello, romper el silencio tampoco será optar por usar el femenino como genérico. No es éticamente justo. La solución es simple y fácil: usar el lenguaje para nombrar nuestro mundo diverso de la manera más enriquecedora posible, sin exclusiones, sin olvidos, sin excusas. Pero este hacer es lo menos común, y no hay que dejar de denunciarlo, pues sus raíces son profundas.

Quizás el Real Decreto 1494/2011 no nombra a las maestras porque se sigue utilizando la terminología validada por el Diccionario de la lengua española de la Real Academia Española (2014). La edición del tricentenario de este diccionario recoge la siguiente palabra y definición: maestro, tra. Del lat. magister, -tri; la forma f., del lat. magistra. 5. $\mathrm{m}$. y f. Maestro de primera enseñanza. De forma específica se menciona a la maestra para hacer alusión a: 23. f. coloq. p. us. Mujer del maestro. 24. f. desus. Mujer que enseña a las niñas en una escuela o colegio. Otras acepciones que recoge el diccionario de la lengua española, que podría interpretarse hacen referencia también a las maestras son, por ejemplo: $4 . \mathrm{m}$. y f. Persona que enseña una ciencia, arte $\mathrm{u}$ oficio, o tiene título para hacerlo. 6. m. y f. Persona que es práctica en una materia y la maneja con desenvoltura.

El término en masculino se da como válido en algunas acepciones y prevalece para nombrar un colectivo que es plural, negando de esta forma la diferencia sexual y el derecho de cada persona a ser reconocida en su oficio y visibilizada en su singularidad. Dicha resistencia no sólo empobrece el lenguaje, sino que perpetúa un mundo desigual donde las mujeres no parecen contar ni tener el mismo valor meritorio para ser nombradas, o si lo son es en referencia al hombre o en ámbitos que se definen como femeninos.

Los cambios se suceden lentamente y parece que aún perdura en este diccionario lo que ya hace más de una década se establecía en las conclusiones del estudio De mujeres y diccionarios. Evolución de lo femenino en la $22^{a}$ edición del DRAE. Las autoras del 


\section{Carrillo}

estudio concluían que "las palabras reflejan el modo de pensar, los usos y los prejuicios de quienes elaboran el diccionario en la Real Academia”, y que quienes redactan el diccionario "deberían cuidar especialmente sus definiciones y el empleo de su lenguaje", para que no refleje la subjetividad del autor o de la autora del texto. Añadían que, para evitar este peligro,

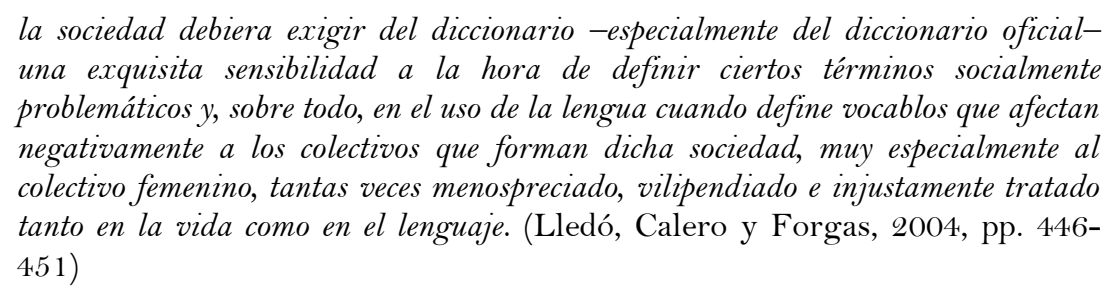

Las consideraciones anteriores respecto al lenguaje son un ejemplo de las contradicciones entre la igualdad formal y la igualdad material, un problema aún no resuelto. Esta es una verdad que la formación tiene la obligación de dar a conocer movilizando su denuncia, al mismo tiempo que desde la propia formación se cambia su uso, es decir, se hace acción de compromiso con la tan reclamada exquisita sensibilidad en el uso del lenguaje, sabiendo que esta acción si bien es significativa aún no será suficiente. Son necesarias las leyes de igualdad, como también son necesarias las prácticas de igualdad. Pero, con todo, las desigualdades entre mujeres y hombres persisten en contextos en los que se siguen viviendo relaciones de abuso de poder y violencia machista.

No silenciar, sino denunciar el uso de un lenguaje sexista que es excluyente y que mantiene la propia normativa, es responsabilidad social de las universidades que quieren contribuir a formaciones justas.

\subsection{Evaluar los impactos de género del lenguaje}

La Plataforma de la Convención sobre la Eliminación de Todas las Formas de Discriminación contra la Mujer (CEDAW) Sombra España 2014, en su Informe Sombra del período 2008-2013 relativo a la aplicación de la CEDAw, concluye que la igualdad de género en España tiene resultados negativos dado los altos índices de discriminación en la legislación y en la práctica. En educación se constata la perpetuación de los estereotipos en los planes de estudio y en los libros de texto; una baja atención a las políticas de integración para la educación de las niñas de etnia gitana; la desaparición de la educación para la ciudadanía y los derechos humanos como asignatura obligatoria; el no fomento de investigaciones sobre las causas de la persistencia de estereotipos de género, así como las insuficientes acciones para combatirlos en los medios de comunicación.

El informe pone de manifiesto que en los países que han ido transitando hacia la democracia, como España, se han producido movimientos, pero éstos no siempre han tenido un calado profundo y permanente. No se pueden negar los cambios que han permitido avanzar en la igualdad entre mujeres y hombres, pero en las sociedades del presente se observa que allí donde se habían dado pasos, éstos no han sido suficientes y, con excusa de las nuevas crisis, disminuyen su ritmo e incluso se dan pasos de retroceso. La igualdad, a nivel global, aún se encuentra a su paso con barreras difíciles de pasar.

En el contexto europeo de forma reiterada se habla de los países nórdicos y de sus políticas de igualdad, y Suecia se pone como país modelo para evidenciar los resultados 
de dichas políticas. La realidad de este país en el 2017 es un gobierno paritario; un parlamento en el que casi la mitad de escaños son ocupados por mujeres; una tasa de empleo de las mujeres de un $78 \%$; hombres y mujeres comparten la crianza de hijas y de hijos; y muchas empresas aplican sistemas de cuotas para las mujeres con objeto de equilibrar el poder, entre otras prácticas orientadas a la igualdad. Un reciente reportaje recoge estos datos, pero también se interroga si realmente el país es un paraíso de la igualdad dado que presenta una de las mayores tasas de violencia machista de la Unión Europea (Blanco, 2017). Si bien en Suecia los indicadores jurídicos para medir las violencias establecen umbrales diferentes a otros países, hay que preguntarse por qué las políticas desarrolladas no desatan los nudos de género que no son sólo nudos de discriminación, de desventaja, de cuotas, de paridad, sino nudos relacionales que mantienen los abusos y las agresiones hacia las mujeres.

Cualquier política, cualquier acción, debería incorporar el impacto de género como criterio de evaluación que mide las consecuencias positivas y negativas de una intervención en las relaciones que se establecen entre mujeres y hombres. Tal criterio debe evaluarse no sólo cuando se han considerado las cuestiones de género, también cuando éstas no han sido explícitamente definidas, pues lo importante es determinar la reproducción de las discriminaciones que viven las mujeres. Junto a ello hay que ponerse lentes de género al evaluar, lentes que permiten ver que las acciones afectan de manera distinta a mujeres, y a hombres (Murguialday, Vázquez, González, 2008).

Considerar el impacto de género es transferible a la educación con la finalidad de analizar este impacto tanto en las políticas como en las prácticas educativas. En la formación de educadoras y educadores aprender a evaluar con lentes de género va a permitir tomar conciencia de la persistencia de problemas aún sin resolver, como es ejemplo el uso de los lenguajes. Este problema, junto a otros, requiere de evaluaciones que permitan introducir cambios, es decir, proyectar educaciones transformadoras orientadas a revertir la tendencia a mantener la diferencia sexual atada a las funciones que establecen las normativas del género, usando el poder que tienen las palabras para ello.

Los centros universitarios han de asumir la responsabilidad social, que ha de ser ética, de un hacer orientado a eliminar, no sólo reducir, la brecha entre los discursos y las prácticas. Este ha de ser el compromiso de toda la comunidad educativa. Compromiso que requiere desaprender la forma de nombrar el mundo para aprender otras formas plurales de nombrar, sabiendo que "somos palabras", que "somos lo que decimos y hacemos al decir", que "somos lo que nos dicen y nos hacen al decirnos cosas con las palabras", que "lo lingüístico es político", y es por ello que se hace necesaria "una ética democrática del lenguaje que favorezca la equidad" (Lomas, 2016, pp. 56-61). Hay que desaprender la forma de ver la realidad y de usar las palabras, para aprender a reconocer y valorar la pluralidad de la misma usando un lenguaje inclusivamente diverso.

Este el reto de las universidades en entornos de resistencias, de nudos del género no resueltos que requieren el esfuerzo de un hacer a contra corriente para seguir otros caminos educativos, quizás no habituales, pero que van a permitir ir trazando la igualdad entre mujeres y hombres, sabiendo que sin igualdad no es posible la libertad, y que tanto una como otra necesitan de la complicidad solidaria en el hacer relacional. Las universidades no pueden vivir al margen de esta evidencia, pues obviarla es negar el enfoque de derechos humanos en la formación. Una opción que no es ética ni deseable. 


\title{
4. El canon de género, negador de la educación de las niñas
}

\author{
Cada siete segundos una niña menor de 15 años contrae matrimonio. \\ De las mujeres que habitan hoy el mundo, más de 700 millones se han casado antes \\ de los 18 años y una de cada tres de esas mujeres se casaron antes de la edad 15.
}

Se estima que 30 millones de niñas corren el riesgo de ser víctimas de una mutilación genital femenina en la próxima década.

Las adolescentes son más propensas a sufrir actos violentos como la violencia sexual.

2,6 mil millones de niñas y mujeres viven en países donde la violación dentro del matrimonio no está tipificada como un delito.

La mortalidad materna es la segunda causa principal de muerte en las adolescentes de 15 a 19 años de edad (después del suicidio).

Cada año 2,5 millones de niñas menores de 16 años dan a luz.

Cuando los recursos del hogar son limitados, las normas sociales en muchos lugares dictan que se debe dar prioridad a los niños en educación y alimentación.

Las mujeres y las niñas son mucho más vulnerables en situación de conflicto y desastre natural.

Por ejemplo, como resultado de los mecanismos negativos de supervivencia, durante el brote de Ébola en Sierra Leona, se calcula que más de 14.000 adolescentes quedaron embarazadas.

Una de cada cuatro adolescentes refugiadas sirias es obligada a casarse.

Muchas niñas, en busca de una vida mejor, acaban siendo engañadas y empujadas a realizar trabajos forzados o con fines de explotación sexual. (Lenhardt et al., 2016, p. 5).

En el Informe 2016 del Programa de las Naciones Unidas para el Desarrollo (PNUD) se afirma que el desarrollo humano consiste en ampliar las libertades de todos los seres humanos, y que para ello hay que velar por el desarrollo de las capacidades y las oportunidades de todas las personas sin exclusiones. Se proclama que sin mujeres no hay desarrollo, sin embargo, los datos del informe indican que son las mujeres las que sufren más discriminaciones, es decir, son el grupo en desventaja más numeroso. Empobrecidas, precarizadas, relegadas, ellas siguen viviendo los nudos del género. Ellas siguen siendo excluidas del derecho a tener derechos.

\subsection{La educación, un derecho de las niñas}

En el ámbito de la educación el informe de la UNESCO (2015) Género y la EPT 2000-2015 concluye que el nacer niña continúa siendo el gran obstáculo para que sea reconocido el derecho a tener derechos. Los contenidos del informe ponen de manifiesto que menos de la mitad de los países logran la paridad de género en la educación. Se indica que se han producido avances de paridad en la enseñanza primaria y secundaria, pero a 62 millones de niñas en el mundo se le niega el derecho a la educación. El informe remarca el hecho que las niñas siguen teniendo más dificultades para realizar los estudios de primaria y casi la mitad de las que están sin escolarizar nunca asistirán a la escuela, es decir, 15 millones de niñas en comparación con la tercera parte de los niños que están en esta situación. Es alarmante también la ausencia de progreso en la alfabetización de las mujeres adultas, dos tercios de las personas adultas analfabetas son mujeres. La violencia de género en los contextos educativos, familiares y sociales, los matrimonios precoces, el 
empobrecimiento, las guerras, las vivencias de migración, entre otros problemas graves, son barreras que impiden la educación a las niñas. Las desigualdades persisten en el seno de los propios países y al mismo tiempo entre países.

La agenda de los Objetivos de Desarrollo Sostenible para el 2030 plantean el logro de 17 objetivos. Entre ellos el Objetivo 4 hace referencia a lograr una educación inclusiva y de calidad, y el Objetivo 5 remite a la igualdad de género para poner fin a la discriminación de las mujeres y de las niñas. Objetivos que sería deseable no se quedaran en discurso por falta de voluntad política y tuvieran que formar parte de nuevas agendas por no haberse cumplido. En este sentido, Save the Children afirma que, si realmente se quiere cumplir con el compromiso de no dejar a nadie atrás, habrá que preocuparse y actuar para revertir la situación de discriminación y exclusión de las niñas. En su informe de 2016, Every last girl. Free to olive, free to learn, free from harm, se insiste en éste objetivo que debe ser central, puesto que la realidad de lo datos es tal que muchas personas prefieren no verlos. Ejemplos son los que se incluyen al inicio del apartado que sacan a la luz problemas complejos, de causas múltiples, entre ellas la persistencia de las normativas del género que vulneran los derechos humanos de las niñas e impactan en su educación precarizándola, interrumpiéndola o negándola totalmente.

\subsection{Proyectar desde la formación universitaria el derecho a la educación}

La formación inicial, pero también permanente, de educadoras y educadores no sólo debe ser sensible a tales realidades, hablar de ellas, sino posicionarse éticamente ante las mismas, siendo consciente que las desigualdades se reproducen y se resisten a desaparecer bajo políticas educativas que se olvidan de las niñas. Posicionarse éticamente es necesario porque las transformaciones profundas lo serán cuando se produzcan cambios en las normativas que no permitan la negación de derechos y cuando estos cambios sean traducidos en las concepciones y en las prácticas. Son estos cambios el resultado de verdaderas revoluciones en la razón y en el corazón que llevan a la conquista de derechos "para los más débiles que han sido limitados por los más fuertes". Revoluciones que van acompañadas del convencimiento que "cada progreso de la igualdad, ha nacido siempre al desvelar una violación de la persona que se ha convertido en intolerable" (Ferrajoli, 2008, p. 52).

El reconocimiento de la educación como Derecho Humano, también para las niñas, es esencial para que la educación no dependa del mercado libre ni esté determinada por el poder adquisitivo de las personas. Esto fue puesto de manifiesto por Katarina Tomasevski (2004), en calidad de Relatora Especial del Derecho a la Educación de las Naciones Unidas. En el Informe 2004 Economic, social and cultural rights. The right to education, ponía de manifiesto un cambio en el vocabulario que persiste en el presente. La expresión derecho a la educación se substituyó por acceso a la educación, y la obligación de los gobiernos de velar para que como mínimo la enseñanza obligatoria fuera gratuita se atenuó poniendo la palabra entre comillas - gratuita-. La autora denunció que estas variaciones lingüísticas tenían la intencionalidad de reconocer que había que financiar la educación, pero negando implícitamente que ello debiera hacerse con cargo a recursos públicos. La privatización de la educación repercute de forma grave en la educación de las niñas y, como consecuencia, debilita sus otros derechos.

La educación es la puerta de los demás derechos, pero para darse plenamente hay que garantizar que sea asequible, es decir obligatoria y gratuita, siendo los gobiernos los responsables de que esto sea así. Sobre la gratuidad habrá que superar las políticas 


\section{Carrillo}

débiles que limitan la misma a la enseñanza obligatoria. Si la educación es derecho humano, deberá ser asequible de forma permanente, a lo largo de la vida. Para ello hay que atender de forma específica al hecho que la asequibilidad supone un problema mayor para las mujeres empobrecidas y precarizadas en sus vidas.

Junto a la asequibilidad hay que garantizar la accesibilidad, pero no se trata sólo de acceder, sino de la equidad en el acceso. Hay que analizar la realidad de las desigualdades para descubrir que no se accede a centros con las mismas condiciones, y que las niñas continúan encontrándose con los obstáculos del sexismo que hacen más difícil su acceso. A qué se accede, cómo se accede y qué obstáculos hay que eliminar son elementos a tener en cuenta cuando se habla de acceso, que no debe ser sólo acceso a una escuela, sino acceso a la educación.

En este sentido, el derecho a la educación remite también a calidad de los centros, de sus recursos, de la formación docente. Calidad que se expresa cuando las condiciones de escolarización no están deprimidas, sino que son aceptables porque están cuidadas, al mismo tiempo que también se cuida la atmósfera moral de los centros para que las niñas se sientan bien, formando parte y siendo protagonistas.

La educación no sólo es ocupar un espacio, sino que como derecho también refiere a ser persona reconocida y valorada en el mismo. La adaptabilidad a la diversidad es otro de los contenidos fundamentales de una educación atenta a las biografías personales de las niñas para acompañarlas facilitando su empoderamiento.

La educación como derecho humano, no lo es, cuando alguno de los indicadores referidos -asequible, accesible, aceptable, adaptable- no es vivido, o sólo se aprecia parcialmente. El derecho a la educación, lo es, cuando es universal y es indivisible.

Los datos muestran que la educación no es vivida plenamente por las niñas. En el 2011 las Naciones Unidas declaraban el 11 de octubre Día Internacional de la Niña, un día que se suma al 8 de marzo (día internacional de la mujer) y el 25 de noviembre (día internacional de la eliminación de la violencia contra la mujer), para recordar que la diferencia sexual sigue provocando desigualdades de género y negación de derechos que son de alta gravedad en las niñas. Aunque lo días internacionales pueden resultar un tanto anecdóticos, la formación de educadoras y educadores no debe olvidarlos, al mismo tiempo que promueve, de manera permanente, deseables éticas aplicadas que permitan deshacer los nudos del género y hacer real el derecho a la educación también para las niñas.

\section{Referencias}

Añón, M. J. (2014). Derechos humanos y obligaciones positivas. En J. M. Bernuz y M. Calvo (Eds.), La eficacia de los derechos sociales (pp. 43-71). Valencia: Tirant lo Blanch.

Ayuste, A., Rodríguez, M. C., Vila, E. S. y Valdivieso, S. (2016). Educación y género en la formación docente en un enfoque de equidad y democracia. En I. Carrillo (Coord.), Democracia y educación en la formación docente (pp. 117-140). Vic: Serveis de Publicacions de la UVic-UCC.

Blanco, S. (5 de marzo de 2017). Suecia: ¿paraíso de la igualdad? El País Semanal. Recuperado de http://elpaissemanal.elpais.com/documentos/suecia-laboratorio-la-igualdad/

Camps, V. (1990). Virtudes públicas. Madrid: Espasa-Calpe. 
Carrillo, I. (Coord.). (2016). Relacions de poder, violències $i$ altres formes de relació abusiva. La transversalitat del gènere. Barcelona: Programa de Millora i Innovació en la Formació Inicial de Mestres.

CEDAW. (2014). Informe sombra 2008-2013 sobre la aplicación en España de la convención para la eliminación de toda forma de discriminación contra las mujeres. Recuperado de http://www.mujeresenred.net/IMG/pdf/InformeSombraCEDAW16sep2014.pdf

Connell, R. W. (1999). Escuelas y justicia social. Madrid: Morata.

Cortina, A. (2010). Los valores de una ciudadanía activa. En A. Tallone y B. Toro (Coords.), Educación, valores y ciudadanía (pp. 95-132). Madrid: OEI y Fundación SM.

Chomsky, N. (2001). La (des)educación. Barcelona: Crítica.

Erazo, X. (2011). Presentación. En X. Erazo, G. Ramírez y M. Scantlebury (Eds.), Derechos humanos, pedagogía de la memoria y políticas culturales (pp. 13-19). Santiago: Editorial LOM.

Ferrajoli, L. (2008). Democracia y garantismo. Madrid: Trotta.

Freire, P. (2003). El grito manso. Buenos Aires: Siglo XXI.

Lagarde, M. (2000). Claves feministas para la autoestima de las mujeres. Madrid: Horas y Horas.

Lenhardt, A., Wise, L., Rosa, G., Warren, H., Mason, F. y Sarumi, R. (2016). Every last girl. Free to olive, free to learn, free from harm. Londres: Save The Children.

BOE. (2007). Ley Orgánica 3/2007, de 22 de marzo, para la igualdad efectiva de mujeres y hombres. Recuperado de https://www.boe.es/buscar/act.php?id=BOE-A-2007-6115

BOE. (2011). Real Decreto 1594/2011, de 4 de noviembre, por el que se establecen las especialidades docentes del cuerpo de maestros que desempeñen sus funciones en las etapas de educación infantil y de educación primaria reguladas en la Ley Orgánica 2/2006, de 3 de mayo, de educación. Recuperado de https://www.boe.es/boe/dias/2011/11/09/pdfs/BOE-A-2011-17630.pdf

Lomas, C. (2016). Lo lingüístico es político. Cuadernos de Pedagogía, 465, 56-61.

López, N. y Cameros M. (2009). La cenicienta que no quería comer perdices. Barcelona: Planeta.

Lledó, E., Calero, A. y Forgas, E. (2004). De mujeres y diccionarios. Evolución de lo femenino en la $22^{a}$ edición del DRAE. Madrid: Instituto de la Mujer.

MECD. (2015). Enseñanzas no universitarias. Estadística del profesorado y otro personal. Curso 20142015. Recuperado de http://www.mecd.gob.es/servicios-al-ciudadanomecd/estadisticas/educacion/no-universitaria/profesorado/estadistica/2014-2015Ultimos-RD.html

Morin, E. (2001). Tenir el cap clar. Per organitzar els coneixements $i$ aprendre a viure. Barcelona: La Campana.

Murguialday, C., Vázquez, N. y González, L. (2008). Evaluación del impacto de género. Barcelona: Cooperacció.

Olivera, M. (2004). De sumisiones, cambios y rebeldías. Mujeres indígenas de Chiapas. Chiapas: Universidad Autónoma de Chiapas.

ONU. (2015). Objetivos de desarrollo sostenible. Recuperado de http://www.un.org/sustainabledevelopment/es/objetivos-de-desarrollo-sostenible/

Pérez, A. E. (2006). La tercera generación de derechos humanos. Pamplona: Aranzadi. 
PNUD. (2016). Informe Desarrollo Humano 2016. Desarrollo humano para todos. Recuperado de http://www.undp.org/content/undp/es/home/librarypage/hdr/2016-humandevelopment-report.html

RAE. (2014). Diccionario de la lengua española. Recuperado de http://dle.rae.es/

Rivera, M. M. (2005). La diferencia sexual en la historia. Valencia: PUV.

Rodríguez, M. E. (2011). Claves para entender los nuevos derechos humanos. Madrid: Los Libros de la Catarata.

Tomasevski, K. (2004). Economic, social and cultural rights. The right to education. Recuperado de http://www.ohchr.org/EN/Issues/Education/SREducation/Pages/AnnualReports.aspx

Una. (2016). Una entre muchas. Bilbao: Astiberri.

UNESCO. (2015). Género y la EPT 2000-2015. Logros y desafíos. Recuperado de http://unesdoc.unesco.org/images/o023/002348/234809S.pdf

Valcárcel, A. (2008). Feminismo en el mundo global. Madrid: Cátedra.

Xarxa Vives. (2017). La perspectiva de gènere en docència i recerca a les universitats de la Xarxa Vives. Situació actual i reptes de futur. Recuperado de http://www.vives.org/PU3.pdf

\section{Breve CV de la autora}

\section{Isabel Carrillo Flores}

Doctora en Pedagogía y Postgrado en Cooperación al Desarrollo. Profesora titular del Departamento de Pedagogía de la Facultad de Educación y Ciencias Humanas de la Universidad de Vic-Universidad Central de Cataluña. Docencia en el Grado de Maestra y Maestro de Educación Infantil y Primaria; Grado de Educación social; Máster Interuniversitario de Estudios de Mujeres y Género. Investigadora del Grupo de Investigación Educativa (2014 SGR 1291) y del Centro de Estudios Interdisciplinarios de Género. Ha dirigido el Departamento de Pedagogía y la Cátedra UNESCO Mujeres, Desarrollo y Culturas. Ha coordinado el Proyecto de Cooperación Educativa con Centroamérica. En la actualidad coordina el Grupo de Trabajo de Género del Programa MIF y participa en proyectos de investigación relacionados con democracia, inclusión, renovación pedagógica, formación ética de maestras y maestros. ORCID ID: O000-00031162-8584. Email: isabel.carrillo@uvic.cat 